\title{
Prone Positioning in Moderate to Severe Acute Respiratory Distress Syndrome Due to COVID-19: A Cohort Study and Analysis of Physiology
}

Journal of Intensive Care Medicine 202I, Vol. 36(2) 24I-252

(C) The Author(s) 2020 (c) (i)

Article reuse guidelines: sagepub.com/journals-permissions DOI: $10.1177 / 0885066620980399$ journals.sagepub.com/home/jic

(S)AGE

\author{
Mehdi C. Shelhamer, DO' $\odot$, Paul D. Wesson, PhD², lan L. Solari, MD', \\ Deanna L. Jensen, CRNA', William Alex Steele, PA', Vihren G. Dimitrov, MD ${ }^{3} \oplus$, \\ John Daniel Kelly, MD, MPH, PhD(c) ${ }^{2,4,5}$, Shazia Aziz, MD', \\ Victor Perez Gutierrez, MD ${ }^{3}$, Eric Vittinghoff, PhD $^{2}$, Kevin K. Chung, $M D^{6}$, \\ Vidya P. Menon, MD ${ }^{3}$, Herman A. Ambris, MD ${ }^{7}$, and Sanjiv M. Baxi, MD, PhD, MPH ${ }^{1,2}$
}

\begin{abstract}
Background: Coronavirus disease 2019 (COVID-19) can lead to acute respiratory distress syndrome (ARDS) but it is unknown whether prone positioning improves outcomes in mechanically ventilated patients with moderate to severe ARDS due to COVID-19. Methods: A cohort study at a New York City hospital at the peak of the early pandemic in the United States, under crisis conditions. The aim was to determine the benefit of prone positioning in mechanically ventilated patients with ARDS due to COVID-19. The primary outcome was in-hospital death. Secondary outcomes included changes in physiologic parameters. Fine-Gray competing risks models with stabilized inverse probability treatment weighting (sIPTW) were used to determine the effect of prone positioning on outcomes. In addition, linear mixed effects models (LMM) were used to assess changes in physiology with prone positioning. Results: Out of 335 participants who were intubated and mechanically ventilated, 62 underwent prone positioning, 199 met prone positioning criteria and served as controls and 74 were excluded. The intervention and control groups were similar at baseline. In multivariate-adjusted competing risks models with sIPTW, prone positioning was significantly associated with reduced mortality (SHR $0.6 \mathrm{I}, 95 \% \mathrm{Cl} 0.46-0.80, P<0.005)$. Using LMM to evaluate the impact of positioning maneuvers on physiological parameters, the oxygenation-saturation index was significantly improved during days $\mathrm{I}-3(P<0.0 \mathrm{I})$ whereas oxygenation-saturation index (OSI), oxygenation-index $(\mathrm{Ol})$ and arterial oxygen partial pressure to fractional inspired oxygen $\left(\mathrm{P}_{\mathrm{a}} \mathrm{O}_{2}\right.$ : $\left.\mathrm{FiO}_{2}\right)$ were significantly improved during days 4-7 $(\mathrm{P}<0.05$ for all). Conclusions: Prone positioning in patients with moderate to severe ARDS due to COVID-19 is associated with reduced mortality and improved physiologic parameters. One in-hospital death could be averted for every 8 patients treated. Replicating results and scaling the intervention are important, but prone positioning may represent an additional therapeutic option in patients with ARDS due to COVID-19.
\end{abstract}

\section{Keywords}

coronavirus disease 2019 , acute respiratory distress syndrome, prone position, severe acute respiratory syndrome coronavirus 2 , respiratory failure

\footnotetext{
' Medical Corps and Nursing Corps, United States Air Force, USA

${ }^{2}$ Department of Epidemiology and Biostatistics, University of California, San Francisco, San Francisco, CA, USA

${ }^{3}$ Department of Medicine, Lincoln Medical Center, New York City Health and Hospitals, The Bronx, New York City, New York, USA

${ }^{4}$ Institute of Global Health Sciences, University of California, San Francisco, San Francisco, CA, USA

${ }^{5}$ F.I. Proctor Foundation, University of California, San Francisco, San Francisco, CA, USA

${ }^{6}$ Uniformed Services University of the Health Sciences, Bethesda, MD, USA

${ }^{7}$ Division of Physical Medicine and Rehabilitation, Lincoln Medical Center, New York City Health and Hospitals, The Bronx, New York City, New York, USA
}

Received August 14, 2020. Received revised November 03, 2020. Accepted November 23, 2020.

Corresponding Author:

Mehdi C. Shelhamer, DO, NYC Health and Hospitals / Lincoln Medical Center, 234 East 14th Street, Suite 8-20, Bronx, New York, NY I045I, USA.

Email: mcshelhamer@gmail.com 
Journal of Intensive Care Medicine 36(2)

\section{Background}

Severe acute respiratory syndrome coronavirus 2 (SARSCoV-2), the cause of coronavirus disease 2019 (COVID-19), has had a profound impact on global public health. The ongoing COVID-19 pandemic has presented numerous clinical management challenges further compounded by overwhelmed health systems. The initial critical care experience in Hubei province, and more broadly in China, inadequately informed preparations for what has been seen in Europe and North America. ${ }^{1}$ Healthcare providers have therefore continued to incorporate and evaluate interventions in real-time. In the setting of critical COVID-19 illness, SARS-CoV-2 infection often results in severe pneumonia and hypoxemia with many patients developing acute respiratory distress syndrome (ARDS). ${ }^{2}$ Hypoxemic respiratory failure with ARDS has poor outcomes overall and COVID-19 associated ARDS is no exception. ${ }^{3,4}$

Several interventions for ARDS have been evaluated over the last 2 decades. In particular, prone positioning is one of few therapeutic interventions for patients with severe ARDS that has demonstrated improved oxygenation and a survival benefit. ${ }^{5}$ Awake prone positioning outside of the intensive care unit (ICU) is safe and may decrease respiratory rate and improve oxygenation with early application potentially delaying need for intubation in patients with COVID-19. ${ }^{6-8}$ In the ICU setting, prone positioning of patients receiving non-invasive ventilation or high-flow nasal canula, with or without sedation, may also be beneficial. ${ }^{8}$ Physiologically, prone positioning may improve matching of ventilation and perfusion, but studies have not linked physiologic changes to clinical outcomes, especially in COVID-19., ${ }^{9,10}$

The South Bronx is a socioeconomically disadvantaged borough in New York City (NYC) that had the highest per capita COVID-19 case count in the United States at 2941 per 100,000 residents with very high hospitalization and death rates. ${ }^{11,12}$ The pressing challenge that COVID-19 brought to NYC necessitated external support through the United States Departments of Defense and Homeland Security, re-distribution and up-training of local hospital staff, support from clinical volunteers, and augmentation through healthcare worker staffing agencies. Given the high volume of critically-ill patients admitted to the hospital, a multidisciplinary team was assembled to provide prone positioning given the support for the practice in other populations with ARDS.

We sought to determine if patients on mechanical ventilation with moderate to severe ARDS who underwent standardized prone positioning had lower mortality and improved within-person physiologic changes. As we rapidly evaluate drugs and interventions for COVID-19, it is crucial to understand if serial prone positioning could be a complementary therapeutic intervention for the most critically ill.

\section{Methods}

\section{Study Design}

A cohort design with participants from the peak of hospitalizations for COVID-19 in exposed (prone positioning) and non-exposed (non-prone-positioning) groups. During the COVID-19 pandemic, much of the hospital was converted into make-shift intensive care units and virtually all inpatients had confirmed COVID-19. During this time, a multidisciplinary prone team including personnel from the United States Air Force Medical and Nursing Corps, the United States Army, civilian contractors, and hospital occupational and physical therapy was assembled to offer positioning maneuvers which were otherwise rarely done due to crisis operations. Details of the prone positioning process, including peri-maneuver checklists, team size and roles, supplies and team schedule are included in Figure 1. In brief, patients were ideally put in the prone position in the afternoon allowing at least 16 hours in position before returning to supine position the following morning. The prone team included a physician, respiratory therapist, registered nurse, runner, and at least 2 members to safely support patient movements. The respiratory therapist served as the default airway expert except when a physician or advanced practice provider was trained in advanced airway management and, in that case, these providers served as airway expert. The prone positioning team did not assume responsibility to provide medical care for any patients during the study period.

\section{Setting and Participants}

Participants were identified from a single level 1 trauma hospital in the South Bronx, New York City, and were included across all hospital services (medicine, surgery, intensive care). All sequential adult patients $(>17$ years of age) were included if they were intubated, had not undergone prone positioning by others, met criteria for prone positioning, and had confirmed SARS-CoV-2 infection by real-time reverse transcription-polymerase chain nasal swab (Bio-Reference Laboratories, Inc., Elmwood Park, NJ, USA) from March 25 through May 2, 2020. The prone team offered positional services for mechanically ventilated patients who met the following criteria (established a priori): arterial oxygen partial pressure to fractional inspired oxygen $\left(\mathrm{PaO}_{2}\right.$ : $\mathrm{FiO}_{2}$ ) $\leq 150 \mathrm{~mm} \mathrm{Hg}$, positive end-expiratory pressure (PEEP) $\geq 10 \mathrm{~cm}$ of water and $\mathrm{FiO}_{2} \geq 0.6$. Patients with a do not resuscitate order were not explicitly excluded from the study. In addition, continuous venovenous hemodialysis (CVVHD), nitric oxide or extracorporeal membrane oxygen (ECMO) were not available in the facility and intermittent hemodialysis or paralytics were rarely used. The ultimate decision for initiating and discontinuing positional movements was made by the primary team overseeing and coordinating care for each patient. Prone positioning was not mandatory, but was routinely available, 24 hours a day, 7 days a week. The study received institutional review board approval (IRB \# 20-007). The prone positioning service was advertised through critical care (surgical and medical), hospital medicine, and physical medicine and rehabilitation leadership. These services had knowledge of and direct access to every patient who was 


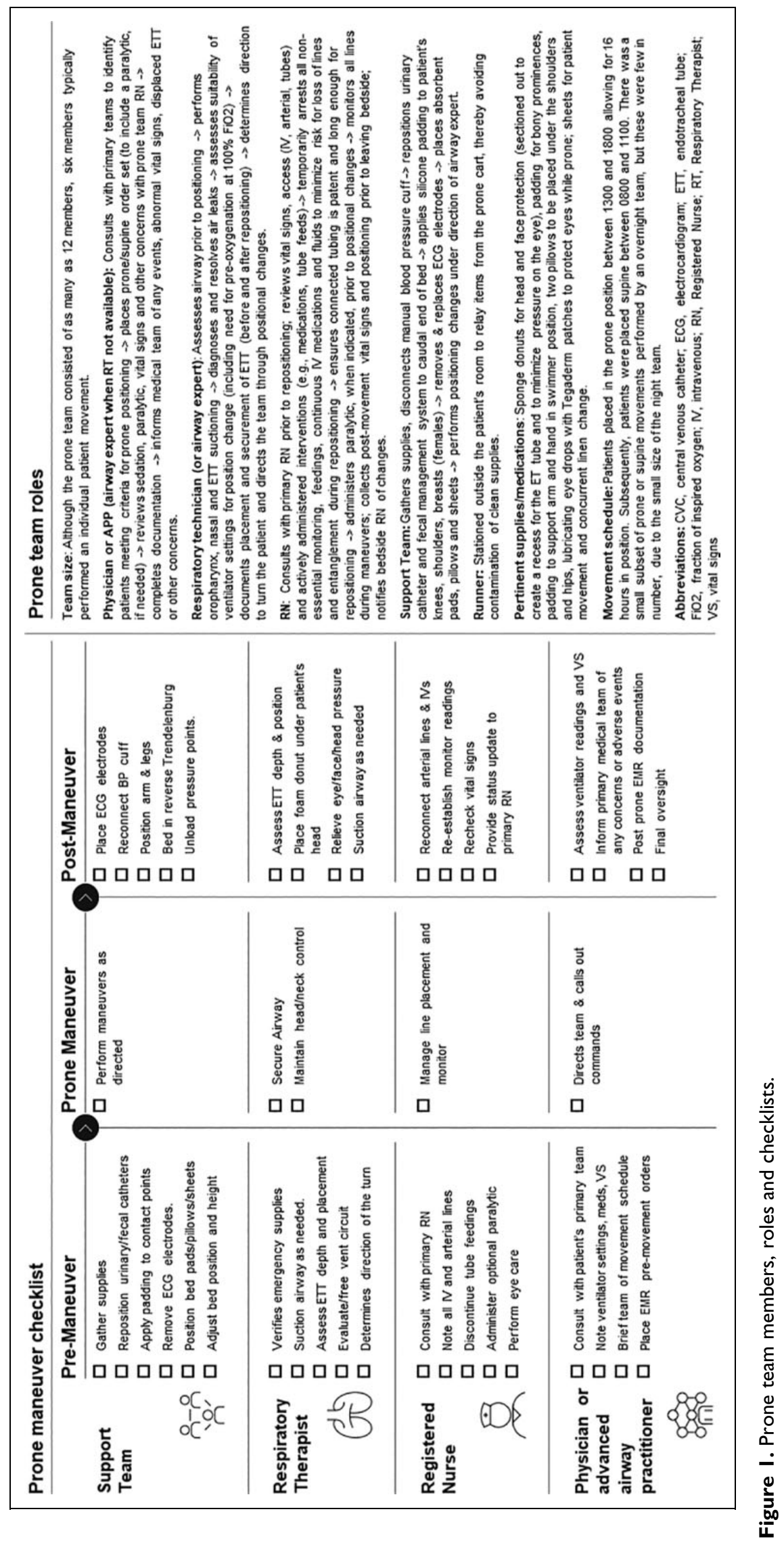




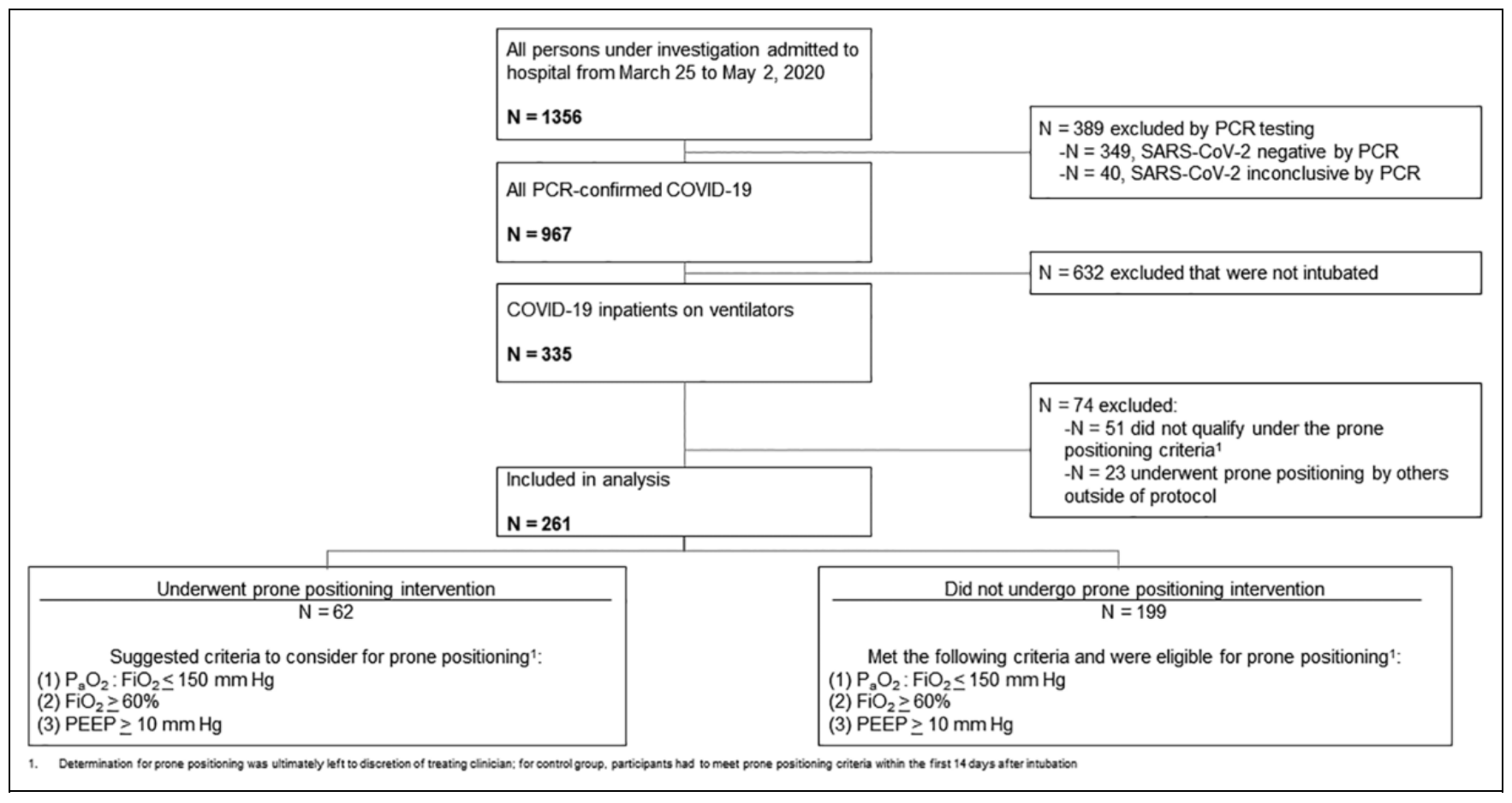

Figure 2. Determination of prone positioning groups during intervention period.

mechanically ventilated in the hospital, even when they were not the primary team.

\section{Measures and Outcomes}

The primary exposure was positional maneuvers, defined as regular alternation between prone and supine positioning. The primary outcomes of interest were in-hospital mortality and, among exposed patients, differences in physiological parameters in prone vs supine position. In the mortality analysis, every patient had at least 30 days elapse following initiation of, or meeting criteria for, prone positioning. Follow-up of unexposed controls began when the participant first met prone positioning criteria during the 2 weeks after intubation. In the analysis of positioning effects on physiologic parameters among exposed patients, repeated measures of the oxygenation index (OI), oxygenation saturation index (OSI), $\mathrm{PaO}_{2}: \mathrm{FiO}_{2}$ and $\mathrm{SpO}_{2}: \mathrm{FiO}_{2}$ were compared during periods of prone and supine positioning. Episodes of positioning separated by more than 48 hours were considered separately. The last physiologic measurement collected in the intervals between each positional change were used in the analysis. After the final positioning change, the last measurement collected within 24 hours was used. Confounders for both analyses were identified based on literature review and directed acyclic graphs. In particular, age, sex, race, body mass index (BMI), acute physiology and chronic health evaluation (APACHE-II) score and vasopressor use were the primary confounders by indication. In the mortality analysis, the APACHE-II score was evaluated at the time of intubation. ${ }^{13}$ BMI and age were categorized for ease of interpretation and clinical utility. The study team obtained the study data through manual electronic medical record chart abstraction (Epic Systems, Verona, WI, USA).

\section{Statistical Analysis}

Characteristics of the cohort were summarized using descriptive statistics as appropriate. Fine-Gray models were used to assess the association between prone positioning and death, accounting for hospital discharge as a competing risk. ${ }^{14}$ Participants remaining in the hospital at the end of follow-up were censored. The proportional sub-distribution hazards assumption was assessed visually through cumulative incidence curves. To minimize confounding by indication, we used standard regression adjustment as well as a doubly robust approach adding stabilized inverse probability treatment weights (IPTWs) to the fully adjusted model. ${ }^{15-17}$ A sensitivity analysis was done to identify changes in results by excluding controls that died within 48 hours of intubation. In addition, number needed to treat was calculated by the inverse of the averaged absolute risk differences at 30 days, for all participants at their actual and counterfactual values of prone positioning, and in combination with their observed covariate values. ${ }^{18}$

Linear mixed models (LMMs) were used to assess the association of prone vs supine positioning with physiologic parameter levels among the exposed. Outcomes were natural log transformed to meet normality assumptions. The LMMs included nested random effects for participant and positioning episode, and allowed for autocorrelation of the residuals. In addition to estimating overall positional effects, we also 
estimated these effects in days 1-3 and 4-7 of each episode. Pearson correlation coefficients were also used to characterize degree of agreement for OI, OSI, $\mathrm{PaO} 2$ : $\mathrm{FiO} 2$ and $\mathrm{SpO} 2$ : FiO2, to support clinical utility in practice. Analyses were performed in Stata (Version 16, StataCorp, College Station, TX, USA).

\section{Results}

During the study period, 335 individuals were intubated and placed on mechanical ventilation. Sixty-two underwent prone positioning while 199 who did not undergo positioning changes, but met criteria to do so, were selected as contemporary controls. Seventy-four individuals were excluded for failing to meet prone positioning criteria or for having undergone prone positioning by providers outside of the standard protocol. A study flow diagram depicts the inclusion and exclusion of participants across groups in Figure 2. Overall, study participants were older, male and mostly self-reported Hispanic or Black. The majority of participants were obese. Diabetes and obstructive lung disease were the most common comorbidities. Most patients were critically ill and septic on admission with a median APACHE-II score at intubation of 17. Most participants required mechanical ventilation at hospital admission (i.e., intubated in the emergency room) and almost all patients $(85 \%)$ received at least some amount of hydroxychloroquine as was consistent with hospital policy during the time. Most patients ultimately expired within 2 weeks. Compared to the control group, the participants who underwent prone positioning were younger (60 versus 66 years old) and were more frequently classified as severe rather than critical on admission to the hospital. Proportions of sepsis on admission and median APACHE-II scores at the time of intubation were similar across groups, but the prone positioning intervention group had less ARDS on admission. Full baseline, demographic and outcome data is summarized in Table 1.

\section{Prone Positioning and Mortality}

Compared to contemporary controls, the prone positioning group had fewer deaths and a longer time to death in those who expired, in spite of similar length of stay and ventilator-free days. Estimates of the association between prone positioning and mortality are summarized in Table 2 . Unadjusted and adjusted competing risks analysis showed that exposed patients were at reduced risk of death (SHR $0.51,95 \%$ CI $0.39-0.66, \mathrm{p}<0.005$ and SHR $0.57,95 \%$ CI $0.42-0.76, \mathrm{p}<0.005$, respectively) compared to unexposed controls. In the doubly-robust analysis adding stabilized IPTWs, inferences were similar (SHR 0.61, 95\% CI $0.46-0.80, p<0.005$ ) and for every 8 patients that underwent prone positioning, one in-hospital death was averted. We found no evidence for violation of the proportional hazards assumption through visual inspection of cumulative incidence curves (Figure 3). Covariate effect estimates are available in Table 3. A sensitivity analysis with removal of controls who died within
48 hours of intubation $(\mathrm{N}=18)$ showed similar results. Regarding adverse events, one dislodged endotracheal tube was noted in a re-positioned patient, but it was thought to have been dislodged prior to the maneuver. One peripheral intravenous line and one peripheral arterial line were inadvertently removed during positioning. Pressure wounds due to positioning were not independently tracked.

\section{Prone Positioning and Physiologic Parameters}

Figure 4 shows the mean trajectories of physiologic parameters over time. Improvements were seen for days 1-3 in the OSI, $\mathrm{P}_{\mathrm{a}} \mathrm{O}_{2}: \mathrm{FiO}_{2}, \mathrm{~S}_{\mathrm{p}} \mathrm{O}_{2}: \mathrm{FiO}_{2}$ and $\mathrm{P}_{\mathrm{a}} \mathrm{O}_{2}$. For days 4-7 of prone positioning, improvement was seen in the $\mathrm{P}_{\mathrm{a}} \mathrm{O}_{2}: \mathrm{FiO}_{2}, \mathrm{~S}_{\mathrm{p}} \mathrm{O}_{2}: \mathrm{FiO}_{2}$ and $\mathrm{P}_{\mathrm{a}} \mathrm{O}_{2}$. Only the $\mathrm{OI}$ failed to show improvement at any time and OSI did not show improvement for days 4-7. During crisis operations with enhanced infection control and use of transport ventilators for routine ventilation, it may be difficult to obtain $\mathrm{P}_{\mathrm{a}} \mathrm{O}_{2}$ and mean airway pressure values and so proxy variables may be helpful. We therefore looked at Pearson correlation coefficients among ratios and indices. Overall, $\mathrm{P}_{\mathrm{a}} \mathrm{O}_{2}: \mathrm{FiO}_{2}$ and $\mathrm{S}_{\mathrm{p}} \mathrm{O}_{2}: \mathrm{FiO}_{2}$ are moderately correlated $(p=-0.51)$, and OSI and OI, and OSI and $\mathrm{S}_{\mathrm{p}} \mathrm{O}_{2}: \mathrm{FiO}_{2}$, are closely correlated ( $p=0.84$ and $p=-0.80$, respectively). The correlations did not differ when split into days 1-3 and 4-7. Results are summarized in Figure 5.

In analyses using LMMs to estimate the association of positioning with physiological indices, 19 of 62 exposed participants contributed more than 1 episode. In these analyses, prone vs supine positioning was significantly associated with overall improvement in $\mathrm{P}_{\mathrm{a}} \mathrm{O}_{2}$ : $\mathrm{FiO}_{2}$ (Table 4). In models allowing positioning effects to differ in days 1-3 and 4-7, prone positioning was associated with improved OSI during days 1-3 $(\mathrm{p}<0.01)$ as well as improved OSI, OI and $\mathrm{P}_{\mathrm{a}} \mathrm{O}_{2}: \mathrm{FiO}_{2}$ during days $4-7$ ( $p<0.05, p<0.01$ and $p<0.001$, respectively). No clear evidence for interaction between positioning and time was found.

\section{Discussion}

We report results from a comprehensive cohort study assessing the potential benefits of prone positioning in COVID-19 patients with moderate to severe ARDS. We found a nearly $40 \%$ reduction in mortality with prone positioning, an effect that appears sustained on cumulative incidence curves. With respect to physiologic parameters, there were meaningful changes across all ratios and indices to suggest that prone positioning is associated with improvements in within-person physiology and that the benefit may persist beyond 3 days. Our findings across both analyses were robust to various adjustments, modifications, sensitivity analyses and nested comparative testing.

Fundamentally, this study has 3 key findings. First, we demonstrated a mortality benefit with prone positioning with a number needed to treat of 8 . The durability of the finding is important, including for a longer time period, and ensuring that 
Table I. Baseline Characteristics, Including Demographic and Clinical Presentation and Outcomes, for All Participants in the Prone Positioning Intervention and Non-Prone Positioning Groups.

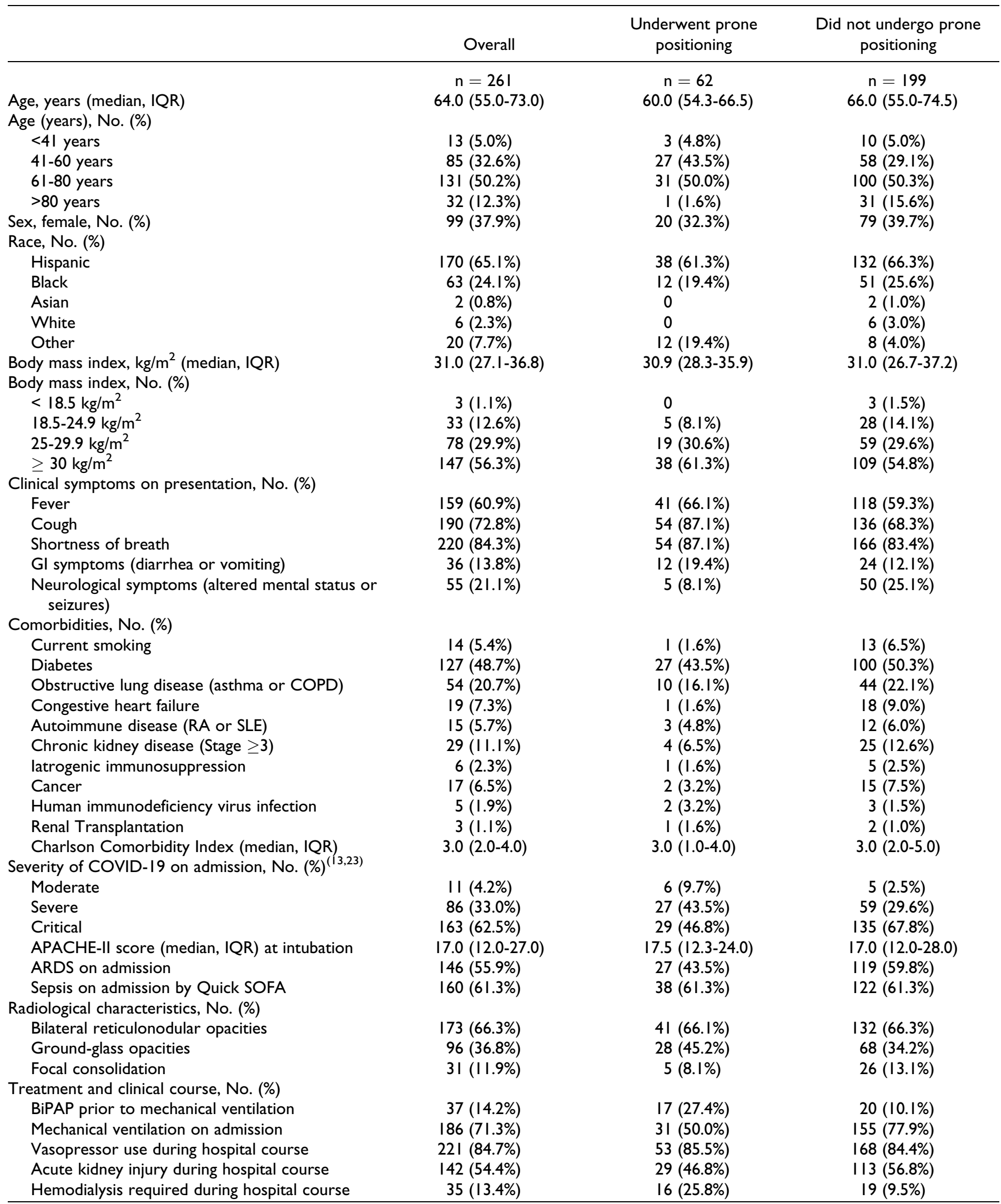


Table I. (continued)

\begin{tabular}{|c|c|c|c|}
\hline & Overall & $\begin{array}{l}\text { Underwent prone } \\
\text { positioning }\end{array}$ & $\begin{array}{l}\text { Did not undergo prone } \\
\text { positioning }\end{array}$ \\
\hline Hydroxychloroquine administered & $219(83.9 \%)$ & $52(83.9 \%)$ & $167(83.9 \%)$ \\
\hline Traditional ICU bed & $86(33.0 \%)$ & $26(41.9 \%)$ & $60(30.2 \%)$ \\
\hline Converted floor ICU bed & $175(67.0 \%)$ & $36(58.1 \%)$ & 139 (69.8\%) \\
\hline \multicolumn{4}{|l|}{ Maneuvers and adjustments } \\
\hline Supine positioning & - & 190 & - \\
\hline Head, neck and shoulder adjustments & - & 443 & - \\
\hline Maneuvers per participant (median, IQR) & - & $4(2-8)$ & - \\
\hline \multicolumn{4}{|l|}{ Outcomes (followed minimum of 30 days), no (\%) } \\
\hline Expired & $215(82.4 \%)$ & $48(77.4 \%)$ & 167 (83.9\%) \\
\hline Discharged & 43 (16.4\%) & $13(21.0 \%)$ & $30(15.1 \%)$ \\
\hline Total extubations & $29(11.1 \%)$ & 7 (II.3\%) & $22(11.1 \%)$ \\
\hline Total re-intubations & $8(3.1 \%)$ & $\mathrm{I}(1.6 \%)$ & $7(3.5 \%)$ \\
\hline Palliative extubations & $10(3.8 \%)$ & $2(3.2 \%)$ & $8(4.0 \%)$ \\
\hline Tracheostomy & $26(10.0 \%)$ & $13(21.0 \%)$ & $13(6.5 \%)$ \\
\hline \multicolumn{4}{|l|}{$\begin{array}{l}\text { Laboratory values on admission, [reference range and } \\
\text { units] reported as median (IQR), } N \text { reported if } \\
\text { different from total }\end{array}$} \\
\hline White blood cell count $\left[4.8-10.8 \times 10^{3}\right.$ microliter] & $9.5(6.9-12.9)$ & $9.5(7.1-12.6)$ & $9.6(6.8-13.1)$ \\
\hline Platelet count [ 150 to 450 per microliter] & $235(|82-30|)$ & $211.5(186-283)$ & $237.0(181-303)$ \\
\hline $\begin{array}{l}\text { Highest d-dimer during hospital course } \\
{[\leq 230 \mathrm{ng} / \mathrm{mill} \text { liliter }]}\end{array}$ & $3543(1163-11838), n=218$ & 3988 (2049.5-13049.8) & $3185(1064-11739), n=156$ \\
\hline
\end{tabular}

ARDS, acute respiratory distress syndrome; BiPAP, bilevel positive airway pressure; COPD, chronic obstructive pulmonary disease; Pro-BNP-N-terminal pro b-type natriuretic peptide; RA, rheumatoid arthritis; SLE, systemic lupus erythematosus.

it can be replicated in other settings will be essential to justify a recommendation, but we have no evidence to attribute the survival benefit in the intervention arm to bias. Second, it appears that there is a benefit to additional days of prone positioning beyond 3 days. The effect seen with 4-7 days of prone positioning may be heavily influenced by a smaller group that realized a differential benefit, but 34 of 89 positioning sequences resulted in at least 4 days of intervention, representing a relatively large proportion of individuals. Third, prone positioning resulted in significant changes in physiologic parameters which may support the underlying hypothesis that prone positioning improves ventilation-perfusion matching. ${ }^{9,10}$ Additionally, we demonstrated the utility of relatively accessible clinical information in the ICU as reasonable surrogates to monitor changes in physiology.
Table 2. Association of a Prone Positioning Intervention and Time to Death by Fine-Gray Competing Risks Analysis.

\begin{tabular}{lccc}
\hline Model & SHR & $95 \% \mathrm{Cl}$ & P-value \\
\hline Unadjusted & 0.51 & $0.39-0.66$ & $<0.005$ \\
Multivariate adjusted & 0.57 & $0.42-0.76$ & $<0.005$ \\
Stabilized doubly robust IPTW & 0.61 & $0.46-0.80$ & $<0.005$
\end{tabular}

Adjusted models control for age, sex, race, body-mass index, Apache II score and vasopressor use.

Our results are consistent with recent multi-center data suggesting a mortality benefit of prone positioning in patients with ARDS whether intubated or not. ${ }^{6-8,19,20}$ There are recommendations for prolonged prone positioning of 12-16 hours daily for mechanically ventilated adult patients with COVID-19 and 


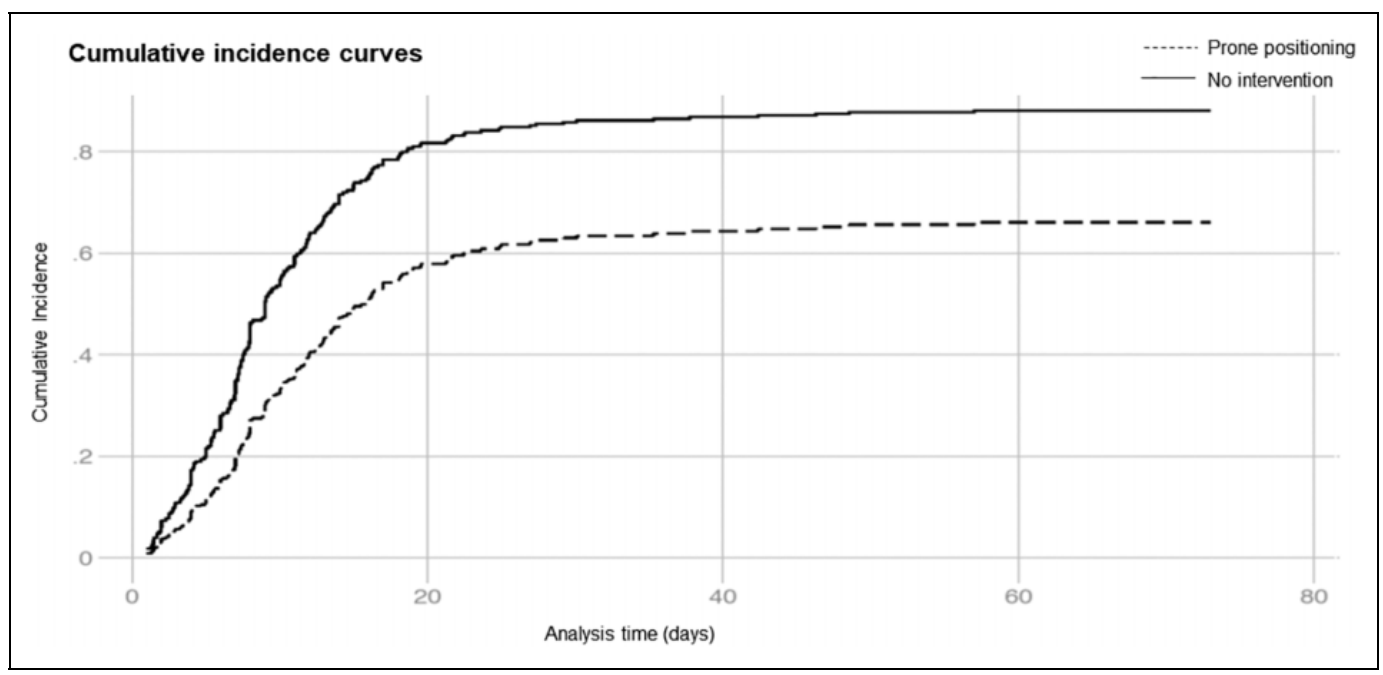

Figure 3. Cumulative incidence curves for participants undergoing prone positioning versus not.

Table 3. Complete Modeling Output for Cox Regression, With Inverse-Probability Treatment Weighting, Adjustments, Stabilized Weights and Accounting for Competing Risks.

\begin{tabular}{|c|c|c|c|}
\hline Variable & SHR & $95 \% \mathrm{Cl}$ & P-value \\
\hline \multicolumn{4}{|c|}{$\begin{array}{l}\text { Prone positioning intervention } \\
\text { (yes vs no) }\end{array}$} \\
\hline No & Reference & - & - \\
\hline Yes & 0.61 & $0.46-0.80$ & $<0.001$ \\
\hline \multicolumn{4}{|l|}{ Age } \\
\hline$<4$ I years & Reference & - & - \\
\hline 4I-60 years & 2.68 & $0.83-8.59$ & 0.10 \\
\hline $61-80$ years & 4.45 & $1.39-14.20$ & 0.01 \\
\hline$>80$ years & 7.11 & $2.13-23.76$ & 0.001 \\
\hline \multicolumn{4}{|l|}{ Sex } \\
\hline Female & Reference & - & - \\
\hline Male & 1.06 & $0.78-1.44$ & 0.69 \\
\hline \multicolumn{4}{|l|}{ Race } \\
\hline White & Reference & - & - \\
\hline Hispanic & 0.33 & $0.18-0.60$ & $<0.001$ \\
\hline Black & 0.38 & $0.20-0.73$ & 0.003 \\
\hline Asian & $*$ & $*$ & $*$ \\
\hline Other & 0.34 & $0.12-0.96$ & 0.04 \\
\hline \multicolumn{4}{|c|}{ Body mass index, No. (\%) } \\
\hline$<18.5 \mathrm{~kg} / \mathrm{m}^{2}$ & $*$ & $*$ & $*$ \\
\hline $18.5-24.9 \mathrm{~kg} / \mathrm{m}^{2}$ & Reference & - & - \\
\hline $25-29.9 \mathrm{~kg} / \mathrm{m}^{2}$ & 0.85 & $0.53-1.36$ & 0.49 \\
\hline$\geq 30 \mathrm{~kg} / \mathrm{m}^{2}$ & 0.87 & $0.57-1.33$ & 0.52 \\
\hline APACCHE-II score & 1.01 & $0.99-1.03$ & 0.26 \\
\hline \multicolumn{4}{|l|}{ Vasopressor use } \\
\hline No & Reference & - & - \\
\hline Yes & 1.18 & $0.76-1.85$ & 0.46 \\
\hline
\end{tabular}

* Observations were dropped from model due to small $\mathrm{N}$ and no variability in treatment (e.g. all within category were treated or all within category were not treated).

refractory hypoxemic respiratory failure, ${ }^{21}$ but the optimal duration of the intervention, its impact on physiologic parameters and details regarding how to organize and structure an intervention team during a crisis have not been completely evaluated. We acknowledge that prone positioning in mechanically ventilated patients is a resource-intensive intervention, particularly in overwhelmed healthcare systems during pandemic conditions. Before adopting prone positioning techniques, staff education and commitment is paramount. If justified by hospitalized patient volume, we recommend identifying personnel and assigning them to a dedicated prone team and tailoring readily available checklists to institutional needs and constraints (Figure 1). ${ }^{22}$

Some limitations of this study should be noted. First, this is a single center retrospective cohort study in a resource constrained environment under crisis operations. As a result, although patients had critical care needs, they were frequently cared for in ad-hoc intensive care units by non-critical care personnel. The decision to initiate or discontinue the intervention under study was left to the treating primary team without defining endpoints. We attempted to address any residual confounding through IPTW and no differences in the results were noted. If the prone team was consulted and the patient had moderate to severe ARDS and met criteria for prone positioning, it was felt that they could benefit from the intervention in addition to lung protective ventilation. Although this was pragmatic for this setting, if prone positioning is implemented elsewhere, the prone teams could consider establishing an opt-out approach with tailored entry and exit criteria, normal cadence of evaluation for candidacy for prone positioning and a mechanism for real-time data capture and quality control assessments. The results of this study may not be readily generalizable to all populations, in particular those with milder disease and those that don't reflect the ethnic diversity seen in the Bronx. The institutional mortality proportion was high $(>75 \%)$ and therefore the impact of the intervention may be attenuated in the setting of advanced interventions (e.g., extracorporeal membrane oxygenation) or the added attention and care of a multidisciplinary team could in and of itself change patient's outcome trajectories, even though they were not involved in care decisions and did not intervene beyond prone 


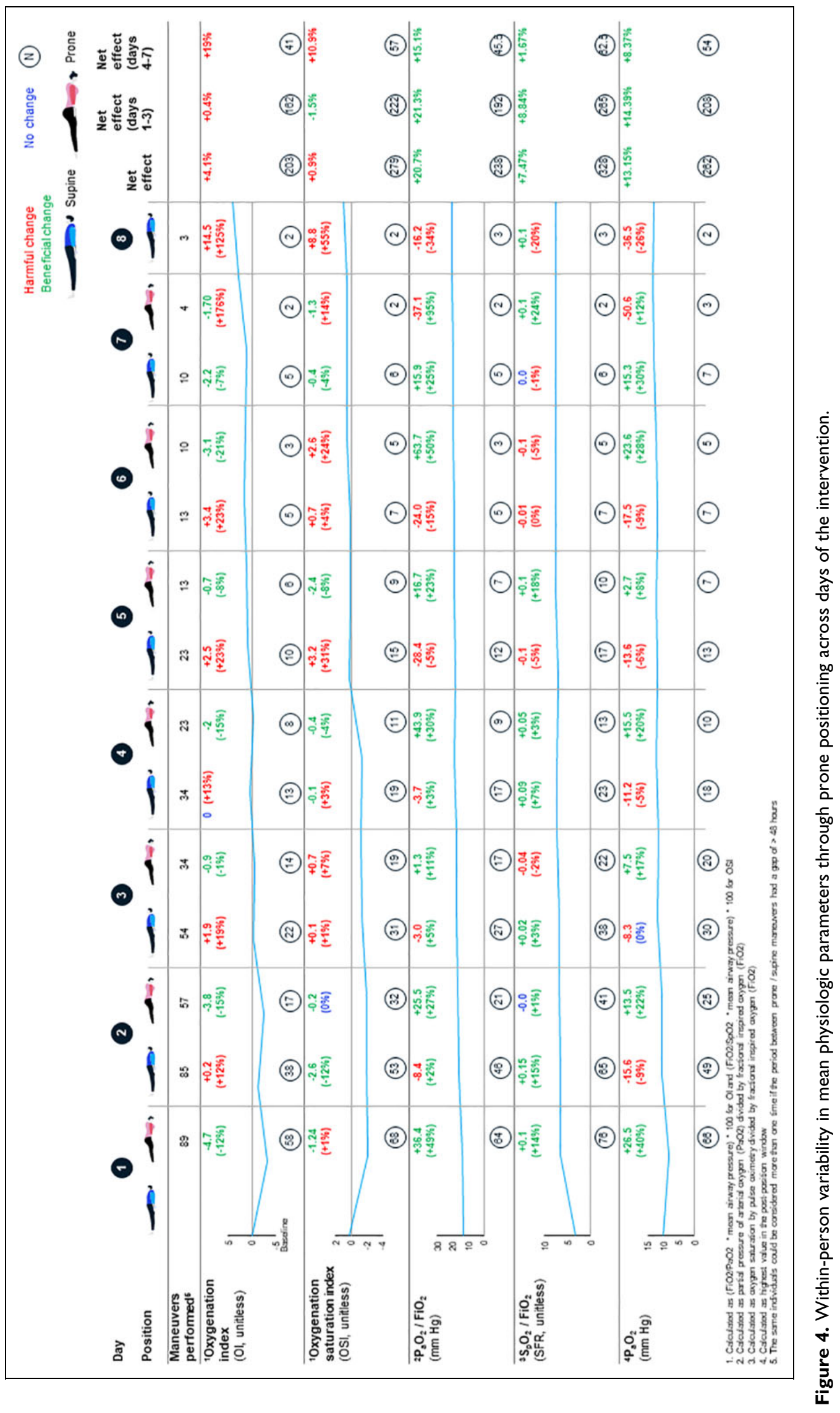




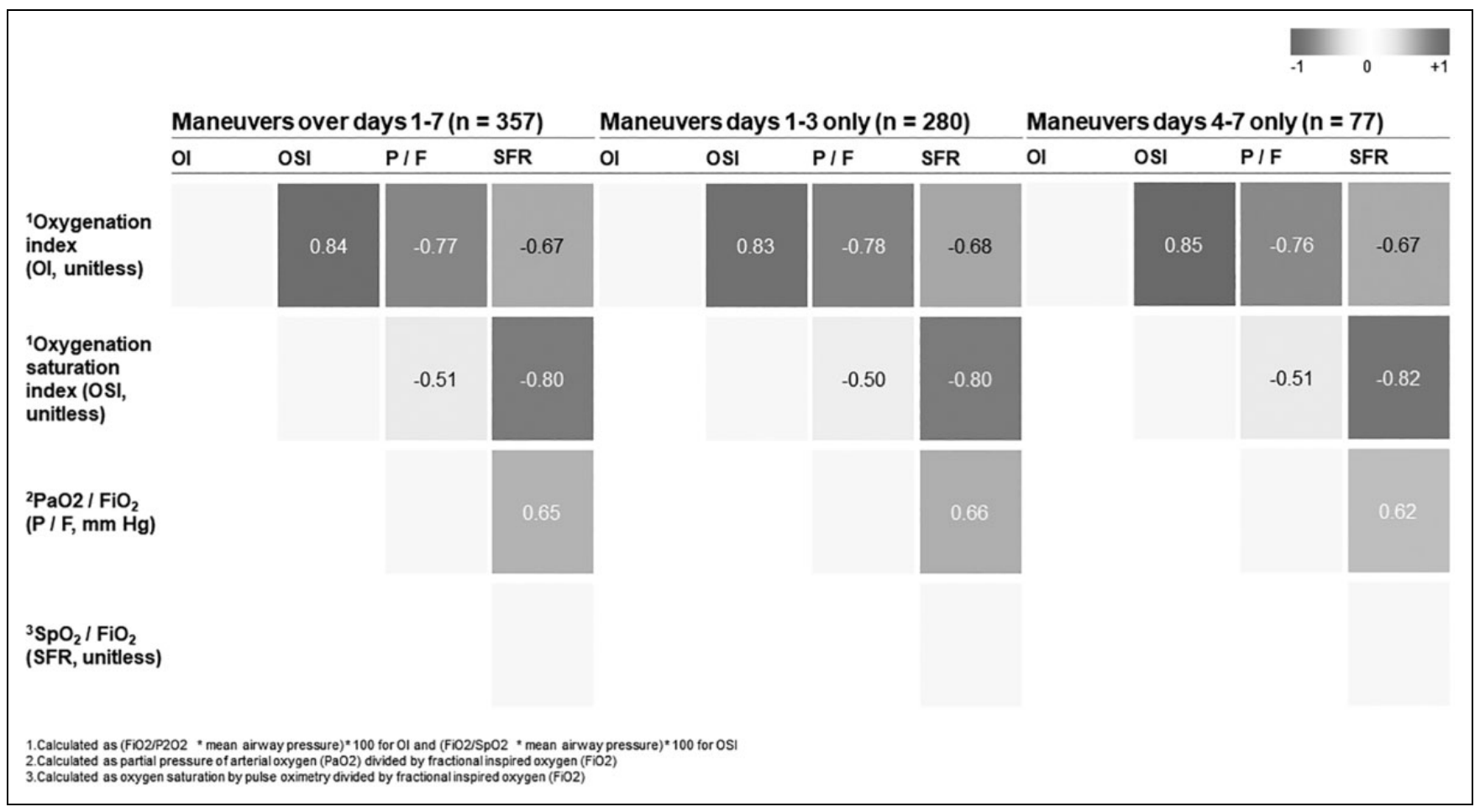

Figure 5. Pearson correlation of physiologic parameters across prone patients split by duration of maneuvers.

Table 4. Adjusted Associations of Prone vs Supine Positioning With Physiological Parameters by Linear Mixed Effects Models.

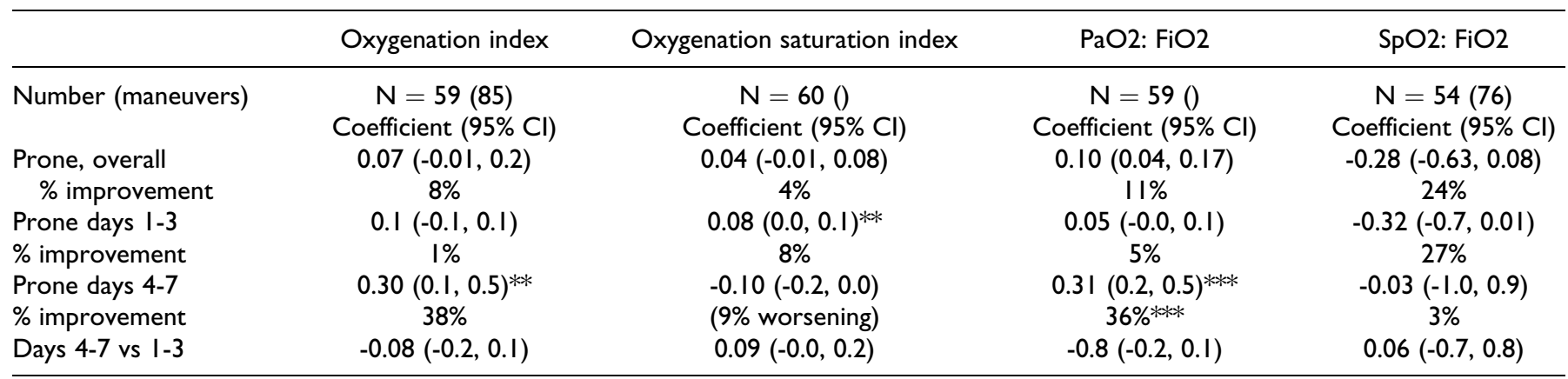

Adjusted for age, sex, race, BMI, Apache II score, and vasopressor use.

$* \mathrm{P}<0.05$, ** $\mathrm{P}<0.01$, *** $\mathrm{P}<0.00 \mathrm{I}$.

positioning. Finally, there may be channeling bias due to disease severity or survivor bias, resulting in lower or higher probability of exposure, respectively. The net result of this could be a bias toward or away from the null. We attempted to address this by ensuring that the populations were comparable at baseline and by systematically including all possible patients. At intubation, there was little difference in the quick SOFA, Charlson Comorbidity index and the APACHE-II scores suggesting that the groups were similar, at least with respect to severity of illness.

There are also some notable strengths of this work. We were able to collect detailed physiologic data in a structured manner to systematically evaluate the impact of the intervention. Also, our population has been gravely understudied in the COVID-19 pandemic and we've been able to contribute significantly to both describing their clinical course as well as critical care interventions for socioeconomically marginalized minority populations. Regarding outcome, we were able to include all patients who would have been eligible for prone positioning as controls creating a sound counterfactual for a contemporaneous comparison of both exposed and unexposed. Finally, compared to existing literature for patients with COVID-19, this study provides results for a large intervention group.

\section{Conclusions}

In summary, we present data supporting prone positioning as an intervention to prolong survival and improve physiologic 
parameters in patients on mechanical ventilation with moderate to severe ARDS due to COVID-19. The findings should be replicated across institutions, but prone positioning may be an important consideration for health systems, particularly in the setting of an evolving suite of complementary interventions in the care of such vulnerable patients.

\section{Abbreviations}

ARDS $=$ Acute Respiratory Distress Syndrome; APACHE-II = Acute Physiology And Chronic Health Evaluation; BMI = Body Mass Index; COVID-19 = Coronavirus Disease 2019; $\mathrm{FiO}_{2}=$ Fraction of Inspired Oxygen; ICU = Intensive Care Unit; IPTWs = Inverse Probability Treatment Weights; LMM = Linear Mixed Models; NYC = New York City; OI = Oxygenation Index; OSI = Oxygenation-Saturation Index; $\mathrm{PaO}_{2}=$ Partial Pressure of Arterial Oxygen; PEEP $=$ Positive End-Expiratory Pressure; SARS-Cov-2 = Severe Acute Respiratory Syndrome Coronavirus 2; sIPTW = Inverse Probability of Treatment Weight; $\mathrm{SPO}_{2}=$ Oxygen Saturation.

\section{Acknowledgments}

We would like to acknowledge the Lincoln Hospital COVID-19 research consortium for supporting various components of data collection (includes Masood Shariff, MD, John Zhang, PhD, Mohammad Aldiabat, MD, Alex Carlos Jr., MD, Sara Tavarez Rodriguez, MD, Bo Yu, MD, Astrid Mendez Batres, MD, Marcia Gossai, MD and Elisenda Valdez, MD). We would also like to thank the NYC hospital system staff, first responders, and healthcare volunteers who came from across the world, and who all made sacrifices to help our patients during a time of great need.

\section{Declaration of Conflicting Interests}

The author(s) declared the following potential conflicts of interest with respect to the research, authorship, and/or publication of this article: The opinions or assertions contained herein are the private views of the authors and are not to be construed as official or as reflecting the views of the Department of the United States Air Force or the Department of Defense.

\section{Funding}

The author(s) disclosed receipt of the following financial support for the research, authorship, and/or publication of this article: This work was supported by National Institute of Allergy and Infectious Disease (K23AI135037 to JDK and K01AI145572 to PW) and National Institute of General Medical Sciences (R01 grant number GM130900 to JDK) of the United States National Institutes of Health.

\section{ORCID iDs}

Mehdi C. Shelhamer, DO (D) https://orcid.org/0000-0003-4374-7377 Vihren G. Dimitrov, MD (D) https://orcid.org/0000-0002-9322-8500

\section{References}

1. Li R, Rivers C, Tan Q, Murray MB, Toner E, Lipsitch M. Estimated demand for US hospital inpatient and intensive care unit beds for patients with COVID-19 based on comparisons with Wuhan and Guangzhou, China. JAMA Netw Open. 2020;3(5):e208297.
2. Murthy S, Gomersall CD, Fowler RA. Care for critically Ill patients with COVID-19. JAMA-J Am Med Assoc. 2020; 323(15):1499-500.

3. Bellani G, Laffey JG, Pham T, et al. Epidemiology, patterns of care, and mortality for patients with acute respiratory distress syndrome in intensive care units in 50 countries. JAMA-J Am Med Assoc. 2016;315(8):788-800.

4. Yang $\mathrm{X}, \mathrm{Yu} \mathrm{Y,Xu} \mathrm{J}$, et al. Clinical course and outcomes of critically ill patients with SARS-CoV-2 pneumonia in Wuhan, China: a single-centered, retrospective, observational study. Lancet Respir Med. 2020;8(5):475-481.

5. Guérin C, Reignier J, Richard JC, et al. Prone positioning in severe acute respiratory distress syndrome. $N$ Engl J Med. 2013;368(23):2159-2168.

6. Caputo ND, Strayer RJ, Levitan R. Early Self-proning in awake, non-intubated patients in the emergency department: a single ED's experience during the COVID-19 pandemic. Kline J, editor. Acad Emerg Med. 2020;27(5):375-378.

7. Carsetti A, Damia Paciarini A, Marini B, Pantanetti S, Adrario E, Donati A. Prolonged prone position ventilation for SARS-CoV-2 patients is feasible and effective. Crit Care. 2020;24(1):1-3.

8. Ding L, Wang L, Ma W, He H. Efficacy and safety of early prone positioning combined with HFNC or NIV in moderate to severe ARDS: a multi-center prospective cohort study. Crit Care. 2020; 24(1):28.

9. Kallet RH. A comprehensive review of prone position in ARDS. Respir Care. 2015;60(11):1660-1687.

10. Koulouras V, Papathanakos G, Papathanasiou A, Nakos G. Efficacy of prone position in acute respiratory distress syndrome patients: a pathophysiology-based review. World J Crit Care Med. 2016;5(2):121.

11. NYC Health. COVID-19: data. 2020 [Internet]. [Accessed 22 May 2020]. https://www1.nyc.gov/site/doh/covid/covid-19-data. page

12. COVID-19 Dashboard by the center for systems science and engineering (CSSE) at Johns Hopkins University (JHU) 2020 [Internet]. Coronavirus COVID-19 Global Cases by the Johns Hopkins University. [Accessed 22 May 2020]. https://corona virus.jhu.edu/map.html

13. Zou X, Li S, Fang M, et al. Acute physiology and chronic health evaluation II score as a predictor of hospital mortality in patients of coronavirus disease 2019. Crit Care Med. 2020;(6):1-9.

14. Fine JP, Gray RJ. A proportional hazards model for the subdistribution of a competing risk. J Am Stat Assoc. 1999;94(446): 496-509.

15. Jonsson Funk M, Westreich D, Wiesen C, Stürmer T, Brookhart M, Davidian M. Doubly robust estimation of causal effects. Am J Epidemiol. 2011;173(7):761-767.

16. Hernán MA, Brumback BA, Robins JM. Estimating the causal effect of zidovudine on CD4 count with a marginal structural model for repeated measures. Stat Med. 2002;21(12): 1689-1709.

17. Robins JM, Hernán MÁ, Brumback B. Marginal structural models and causal inference in epidemiology. Epidemiology. 2000;11(5): 550-560. 
18. De Lemos ML, Barratt AL, Wyer PC, Guyatt G, Simpson JM. NNT for studies with long-term follow-up [1] (multiple letters). Cmaj. 2005;172(5):613-615.

19. Elharrar X, Trigui Y, Dols A-M, et al. Use of prone positioning in nonintubated patients with COVID-19 and hypoxemic acute respiratory failure. JAMA. 2020;323(22):2336-2338.

20. Ziehr DR, Alladina J, Petri CR, et al. Respiratory pathophysiology of mechanically ventilated patients with COVID-19: a cohort study. Am J Respir Crit Care Med. 2020;201(12):1560-1564.

21. COVID-19 Treatment Guidelines Panel. Coronavirus disease 2019 (COVID-19) treatment guidelines. National institutes of health [Internet]. [Accessed 17 May 2020]. https://covid19treat mentguidelines.nih.gov

22. Oliveira VM, Piekala DM, Deponti GN, et al. Safe prone checklist: construction and implementation of a tool for performing the prone maneuver. Rev Bras Ter Intensiva. 2017; 29(2):131-141.

23. Aylward B, Liang W. Report of the WHO-China joint mission on coronavirus disease 2019 (COVID-19). WHO-China Jt mission coronavirus Dis 2019 [Internet]. 2020. Accessed 22 May 2020. https:/www.who.int/docs/default-source/coronaviruse/ who-china-joint-mission-on-covid-19-final-report.pdf 